\title{
Global estimation of anti-malarial drug effectiveness for the treatment of uncomplicated Plasmodium falciparum malaria 1991-2019
}

Giulia Rathmes ${ }^{1 \dagger}$, Susan F. Rumisha ${ }^{1,7^{*}+}$, Tim C. D. Lucas ${ }^{1}$, Katherine A. Twohig ${ }^{1}$, Andre Python ${ }^{1,9}$, Michele Nguyen ${ }^{1}$, Anita K. Nandi', Suzanne H. Keddie', Emma L. Collins' ${ }^{1}$, Jennifer A. Rozier ${ }^{1}$, Harry S. Gibson', Elisabeth G. Chestnutt ${ }^{1}$, Katherine E. Battle ${ }^{1}$, Georgina S. Humphreys ${ }^{2,3,4}$, Punam Amratia', Rohan Arambepola', Amelia Bertozzi-Villa ${ }^{1,5}$, Penelope Hancock' ${ }^{1}$, Justin J. Millar ${ }^{1}$, Tasmin L. Symons ${ }^{1}$, Samir Bhatt ${ }^{6}$, Ewan Cameron 1,7,8, Philippe J. Guerin ${ }^{2,3,4}$, Peter W. Gething ${ }^{7,8}$ and Daniel J. Weiss ${ }^{1,7,8}$

\begin{abstract}
Background: Anti-malarial drugs play a critical role in reducing malaria morbidity and mortality, but their role is mediated by their effectiveness. Effectiveness is defined as the probability that an anti-malarial drug will successfully treat an individual infected with malaria parasites under routine health care delivery system. Anti-malarial drug effectiveness (AmE) is influenced by drug resistance, drug quality, health system quality, and patient adherence to drug use; its influence on malaria burden varies through space and time.

Methods: This study uses data from 232 efficacy trials comprised of 86,776 infected individuals to estimate the artemisinin-based and non-artemisinin-based AmE for treating falciparum malaria between 1991 and 2019. Bayesian spatiotemporal models were fitted and used to predict effectiveness at the pixel-level ( $5 \mathrm{~km} \times 5 \mathrm{~km})$. The median and interquartile ranges (IQR) of AmE are presented for all malaria-endemic countries.

Results: The global effectiveness of artemisinin-based drugs was 67.4\% (IQR: 33.3-75.8), 70.1\% (43.6-76.0) and 71.8\% (46.9-76.4) for the 1991-2000, 2006-2010, and 2016-2019 periods, respectively. Countries in central Africa, a few in South America, and in the Asian region faced the challenge of lower effectiveness of artemisinin-based anti-malarials. However, improvements were seen after 2016, leaving only a few hotspots in Southeast Asia where resistance to artemisinin and partner drugs is currently problematic and in the central Africa where socio-demographic challenges limit effectiveness. The use of artemisinin-based combination therapy (ACT) with a competent partner drug and having multiple ACT as first-line treatment choice sustained high levels of effectiveness. High levels of access to healthcare, human resource capacity, education, and proximity to cities were associated with increased effectiveness. Effectiveness of non-artemisinin-based drugs was much lower than that of artemisinin-based with no improvement over time: 52.3\% (17.9-74.9) for 1991-2000 and 55.5\% (27.1-73.4) for 2011-2015. Overall, AmE for artemisinin-based
\end{abstract}

\footnotetext{
*Correspondence: susan.rumisha@bdi.ox.ac.uk

${ }^{\dagger}$ Giulia Rathmes and Susan F. Rumisha are joint first authors

${ }^{1}$ Malaria Atlas Project, Big Data Institute, Nuffield Department of Medicine, University of Oxford, Oxford, UK

Full list of author information is available at the end of the article
}

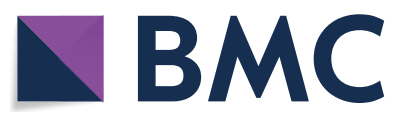

(c) The Author(s) 2020. This article is licensed under a Creative Commons Attribution 4.0 International License, which permits use, sharing, adaptation, distribution and reproduction in any medium or format, as long as you give appropriate credit to the original author(s) and the source, provide a link to the Creative Commons licence, and indicate if changes were made. The images or other third party material in this article are included in the article's Creative Commons licence, unless indicated otherwise in a credit line to the material. If material is not included in the article's Creative Commons licence and your intended use is not permitted by statutory regulation or exceeds the permitted use, you will need to obtain permission directly from the copyright holder. To view a copy of this licence, visit http://creativeco mmons.org/licenses/by/4.0/. The Creative Commons Public Domain Dedication waiver (http://creativecommons.org/publicdomain/ zero/1.0/) applies to the data made available in this article, unless otherwise stated in a credit line to the data. 
and non-artemisinin-based drugs were, respectively, 29.6 and 36\% below clinical efficacy as measured in anti-malarial drug trials.

Conclusions: This study provides evidence that health system performance, drug quality and patient adherence influence the effectiveness of anti-malarials used in treating uncomplicated falciparum malaria. These results provide guidance to countries' treatment practises and are critical inputs for malaria prevalence and incidence models used to estimate national level malaria burden.

Keywords: Falciparum malaria, Anti-malarial drug effectiveness, Drug quality, Global

\section{Background}

The Global Burden of Disease (GBD) study estimated that 619,800 (95\% uncertainty intervals: 440,100$839,500)$ malaria deaths occurred worldwide in 2017 [1], with over $80 \%$ of deaths occurring in sub-Saharan Africa [2]. Malaria mortality has decreased substantially over the last two decades through increased investment in the availability of effective treatments, such as artemisinin-based combination therapy (ACT) and preventive measures such as long-lasting insecticidal nets [3-5]. Anti-malarial treatments are key to curbing malaria burden and mortality as they reduce the individual's risk of severe disease and death in incident cases $[6,7]$ while also decreasing the infectious reservoir of individuals from where mosquitoes can acquire a blood meal. Reaching global malaria reduction targets requires a detailed understanding of current treatment coverage levels as well as factors limiting their effectiveness. Coverage of anti-malarial effectiveness in the population necessitates that: (i) all patients with confirmed malaria infection access an anti-malarial treatment; (ii) the provided drug is of high efficacy and good quality; and, (iii) all patients receive an optimal dosing and adhere to the treatment regimen. This covers all relevant providers involved in managing malaria patients, including private sector, both formal and informal. Whilst efficacy, which drives most of the drug policy decisions, is primarily dictated by the evolution of drug-resistant parasite phenotypes, anti-malarial drug effectiveness (AmE) is a composite measure that encompasses clinical efficacy (the performance of the medicine under controlled conditions) and other real-world clinical practice limitations. AmE is influenced by: (i) patient-specific responses to the treatment, including absorption, genetics, co-morbidities, special conditions such as pregnancy, very young age, or drug-drug interactions; (ii) patient adherence to the drug's use instructions; (iii) healthcare providers' skill, knowledge and prescription practises; (iv) health system performance; (v) access to the healthcare system; (vi) healthcare expenditure; and, (vii) other sociodemographic characteristics that limit the appropriate use of anti-malarial drugs.
The World Health Organization (WHO) recommends the use of ACT for treatment of uncomplicated falciparum malaria [8], as this species causes the most severe forms of malaria and subsequently death. Currently, ACT is used by most malaria-endemic countries and territories $[9,10]$ as first-line treatment for falciparum malaria (Additional file 1: Section 1), and its use has been widespread. For example, $82 \%$ of febrile children treated with an anti-malarial received an ACT medicine in public health facilities within sub-Saharan Africa between 2015 and 2018 [11]. High efficacy of ACT for Plasmodium falciparum infection has been reported in sub-Saharan Africa, with no artemisinin resistance confirmed in this region [12]. Historically, anti-malarial resistance has been a major obstacle in the fight against malaria [3, 12-15]. Chloroquine (CQ) resistance was first observed in Southeast Asia and in South America in the late 1950s, and later spread to Africa. It was replaced by sulfadoxinepyrimethamine (SP), to which resistance quickly emerged and spread from Southeast Asia to most endemic areas, and the drug became ineffective [16]. Evidence supporting the high efficacy and safety of artemisinin derivatives when paired with a partner drug became available in the late 1990s [17-19]. However, the emerging artemisinin resistance reported since the mid-2000s poses a significant threat to the recent gains in malaria control. Resistance to artemisinin was first identified in the Greater Mekong Sub-region (GMS), and there are concerns that it has spread to densely populated countries like India and recently to parts of sub-Saharan Africa [3, 14, 20-30]. The historical use of artemisinin as a monotherapy [12, $14,15]$, or the use of partner drugs with similar modes of action and cross-resistance [31, 32], are among the primary factors fuelling the development of resistance. Other potential factors include the use of sub-standard and falsified medicines, high prevalence of self-treatment, poor adherence to drug use protocols, weak healthcare systems, and unmonitored treated cases [33-37]. All these underscore the need for a full understanding of the spatiotemporal pattern of ACT effectiveness in all $P$. falciparum endemic countries. Likewise, the effectiveness of non-artemisinin anti-malarials remains important as they are still first-line treatments for falciparum 
malaria in some countries [11] and widely used in many areas despite adaptation of WHO recommendations [12]. Together the ACT and non-ACT results from this research will provide crucial information for assessing the impact of effectiveness on falciparum malaria burden, monitoring emerging resistance (including multi-drug resistance), and better characterizing anti-malarial drug distribution, quality, access, and use [17, 37-40]. When it comes to saving lives, effectiveness of drugs used for treatment is a key control intervention, and the need to characterize changing patterns of $\mathrm{AmE}$ is evident [41]. A global assessment of anti-malarial drug efficacy was published in 2010; existence of recently conducted and published anti-malarial efficacy trials [42] emphasizes the rationale for deriving updated global AmE estimates that will inform researchers, stakeholders and countries' malaria control programmes [14, 20, 43-46].

To precisely monitor and compare AmE over space and time, and at a global scale, standardized data and methodologies are required. Clinical efficacy data are a key input for such analyses, and several databases cataloguing this metric have been established. As the databases were created for differing research purposes, they have inconsistent structures and varying foci, including specific drugs, regions or time periods [44, 47-56] (Additional file 1: Section 1). Most of these databases do not provide patient-specific data to support further statistical analysis, and contain location-based drug efficacy estimates derived using different methodologies, in both design and analysis [48]. The WorldWide Anti-malarial Resistance Network (WWARN) responded to the challenge of comparing varying anti-malarial drug efficacy estimates by: (i) acquiring individual-patient-level data from efficacy trials conducted and published since 1960 [57]; (ii) re-analysing the patient-level data using a consistent methodology (modified Intention-To-Treat analysis); and, (iii) using standardized indicator definitions to produce comparable drug efficacy estimates $[58,59]$. The result of WWARN's work is the most comprehensive, standardized and accessible anti-malarial drug efficacy database yet created. Critically, WWARN dataset provides comparable results within and between countries, and over time thus supporting a spatiotemporal analysis.

Clinical drug efficacy trials suffer from various complications, including non-compliance, protocol withdrawals and deviations (e.g., co-morbidity, exposure to new infections and health worker mistakes) that may result in participants being dropped during the analysis phase. The Intention-To-Treat analytical approach is advantageous because it includes all study participants according to the initial randomization, regardless of deviations from the protocol, such as participant withdrawals from the study or re-infection [59-61].
This method gives more conservative and unbiased efficacy estimates that are closer to what would happen in clinical practice and are proxy for effectiveness [62-64] (study endpoints by WHO and WWARN-Additional file 1: Section 1). There are deficiencies in existing routine health information systems for adequately monitoring responses to malaria treatment, and few studies have assessed effectiveness of anti-malarials globally. As such, analysing WWARN estimates within geospatial models that (i) include health system, socio-demographic and environmental factors, while (ii) adjusting for adherence and quality of drugs, provides a reasonable basis for deriving measures of AmE [65]. Furthermore, the covariates introduce information linked to effectiveness rather than efficacy, thereby allowing the model to amplify or reduce the gap between efficacy and effectiveness based on local conditions.

This analysis generates fine-scale, global temporally dynamic maps of AmE for uncomplicated falciparum malaria. This metric defines the treatment success rate of an anti-malarial drug when administered to P. falciparum-infected individuals under typical use conditions (e.g., drugs obtained from facilities or pharmacies). This considers all individuals with parasitological- or clinically confirmed malaria infections that subsequently received an anti-malarial drug for treatment, regardless of whether they had a malaria-attributable fever, a fever attributable to a co-infection, or no fever. Effectiveness is estimated for both artemisinin-based and non-artemisinin-based treatment in all malaria-endemic countries from 1991 to 2019. The AmE models include covariates for health system factors, climate and environmental variables, sociodemographic, malaria transmission risks, and population. The estimates are adjusted for quality of anti-malarial drugs and adherence to dosage regimens $[33,66]$. The rationale for modelling AmE rather than clinical efficacy is threefold: (i) effectiveness is a more relevant metric for assessing anti-malarial impacts when administered within a real-world clinical setting (i.e., after it becomes a front-line treatment for malaria within a country); (ii) modelling effectiveness allows use of spatially varying covariates that can feasibly be related to effectiveness but are unlikely to influence efficacy; and, (iii) effectiveness is an essential input for calculating malaria mortality in the GBD study. The GBD study includes annual, nationallevel estimates of morbidity and mortality attributed to malaria, with accompanying high-spatial-resolution $(5 \times 5 \mathrm{~km})$ maps produced by the Malaria Atlas Project (MAP) $[67,68]$. Prior to use in modelling malaria burden, the results of this research $(\mathrm{AmE})$ are combined with proportional anti-malarial use and treatment-seeking rates to generate estimates of effective treatment with anti-malarials, which are then used for modelling $P$. 
falciparum prevalence and estimating the proportion of cases that are not successfully treated.

\section{Methods \\ Data}

The response data used in this research consisted of 232 anti-malarial drug efficacy studies conducted between 1991 and 2016, comprising 756 treatment arms, and information on 86,776 individuals. Among the studies used for analysis, 203 were extracted from the WWARN database, and comparable metrics were extracted from additional studies $(\mathrm{n}=29)$ obtained through a review of articles assembled in the WWARN clinical trials publication library [61]. For consistency, these additional studies were selected using the same criteria applied when generating the WWARN dataset (i.e., additional studies focused on treatment of falciparum malaria, did not include pregnant women, and used a modified Intention-To-Treat approach). The WWARN database consists of publicly available aggregated results harmonized and summarized from anti-malarial drug efficacy studies, including both artemisinin-based (including artesunate monotherapy) and non-artemisinin-based therapy (Additional file 1: Section 1). Information of the trials included in the analysis is presented in Table 1. Additional file 1: Section 2 illustrates distribution and number of studies by treatment type, country and year.

Table 1 Characteristics of anti-malarial drug efficacy trials

\begin{tabular}{|c|c|c|c|c|}
\hline & Africa & Asia & South America & Total $^{\mathbf{a}}$ \\
\hline Number of ACT-studies (\%) & $133(69.4)$ & $65(28.0)$ & $6(2.6)$ & 204 \\
\hline Number of non-ACT-studies (\%) & $62(100)$ & 0 & 0 & 62 \\
\hline Number of subjects (\%) & $64,553(74.4)$ & $21,168(24.4)$ & $1,055(1.2)$ & 86,776 \\
\hline Study year, range & 1993-2015 & 1991-2016 & 2004-2016 & $1993-2016$ \\
\hline \multicolumn{5}{|l|}{ Age at enrolment (no of studies) } \\
\hline Under 5 years of age & 67 & 2 & 0 & 69 \\
\hline All ages & 128 & 63 & 6 & 197 \\
\hline \multicolumn{5}{|l|}{ Treatment studied (no of treatment arms in efficacy study) } \\
\hline \multicolumn{5}{|c|}{ ACT (including monotherapy) } \\
\hline Artesunate & 21 & 24 & 1 & 46 \\
\hline Artemether-lumefantrine (AL) & 169 & 32 & 2 & 203 \\
\hline Artesunate-amodiaquine (ASAQ) & 94 & 6 & 1 & 101 \\
\hline Artesunate-mefloquine (ASMQ) & 15 & 62 & 3 & 80 \\
\hline Artesunate-sulfadoxine-pyrimethamine (ASSP) & 41 & 47 & 0 & 88 \\
\hline Dihydroartemisinin piperaquine (DHAP) & 50 & 53 & 1 & 104 \\
\hline \multicolumn{5}{|l|}{ Non-ACT } \\
\hline Chloroquine (CQ) & 35 & 0 & 0 & 35 \\
\hline Sulfadoxine-pyrimethamine (SP) & 69 & 0 & 0 & 69 \\
\hline Others & 30 & 0 & 0 & 30 \\
\hline \multicolumn{5}{|l|}{ Efficacy \%, median } \\
\hline ACT (including monotherapy) & 97.9 & 100 & 100 & 99.3 \\
\hline Artesunate & 86.4 & 100 & 100 & 95.5 \\
\hline Artemether-lumefantrine & 97.8 & 97.4 & 100 & 98.4 \\
\hline Artesunate-amodiaquine & 97.8 & 94.9 & 97.1 & 96.6 \\
\hline Artesunate-mefloquine & 99.3 & 99.2 & 100 & 99.5 \\
\hline Artesunate-sulfadoxine-pyrimethamine & 97.7 & 100 & & 98.9 \\
\hline Dihydroartemisinin piperaquine & 98.4 & 100 & 99.2 & 99.2 \\
\hline Non-ACT & 83.1 & & & 83.1 \\
\hline Chloroquine & 61.1 & & & 61.1 \\
\hline Sulfadoxine-pyrimethamine & 91.3 & & & 91.3 \\
\hline Others & 82.3 & & & 82.3 \\
\hline
\end{tabular}

a Some studies assessed both drugs, hence counted twice 


\section{Covariates}

Covariates used for modelling were identified from several procedures. First, publications describing factors influencing treatment failure, effectiveness of antimalarials, quality of anti-malarials, anti-malarial usage, and adherence, were identified with a literature review that followed an established methodology [69]. Second, a comprehensive search of the WWARN clinical trials publication library, which includes all available and published anti-malarial efficacy studies, was performed. Papers were considered if they studied P. falciparum only, did not include pregnant women, used a modified Intention-To-Treat statistical analysis approach, and had a follow-up time of at least 28 days. A total of 733 out of 1178 publications that reported efficacy matched these criteria, and all these were reviewed to extract information on covariates related to anti-malarial efficacy. Third, using similar criteria, an additional 47 papers focusing on malaria risk mapping were identified from publication databases and included for review. This literature review identified factors related to malaria infection, transmission and risk that are relevant when assessing the performance of anti-malarial drugs in real-world settings. Finally, grey literature, including WHO Malaria reports, was examined. The databases searched were PubMed, Embase, the Web of Science library, and Google Scholar. All covariates mentioned as having an influence on antimalarial drug efficacy, malaria treatment effectiveness or malaria infection in the compiled literature were identified. Additional file 1: Section 3-Table S3.1 presents some of literature reviewed, identified factors and their importance to this analysis.

The Institute for Health Metrics and Evaluation (IHME) [70] and MAP [71] provided the covariates used in this analysis [69, 72]. IHME collates and produces country- and annual-level variables, including health system access and metrics characterizing socio-economic status. MAP compiles, maintains and generates gridded global covariates characterizing environmental and climatic conditions, which are primarily derived from temporally dynamic and high-resolution satellite images and include temperature, rainfall, vegetation indices, population, night-time lights, and accessibility to cities [69, 7275]. Data were obtained for 69 factors (Additional file 1: Section 3-Tables S3.2 and S3.3). Six transformations (natural logarithm, reciprocal, squared, cubed, exponential) were applied to all continuous variables to incorporate potential non-linear relationships with the response.

\section{Variable selection procedures}

Following assessment of the collated covariates, 38 out of 53 IHME covariates were dropped as they were unavailable for all countries (e.g., they were only modelled for Africa and not globally). Similarly, 3 out of the 16 MAP covariates were excluded due to incomplete geographical coverage. The selection of the remaining variables was performed separately for artemisinin-based and non-artemisinin effectiveness by fitting generalized linear models with study site-specific random effects. The artemisinin-based and non-artemisinin anti-malarials were modelled separately because (i) the performance of the drug classes had markedly different temporal effectiveness patterns, and (ii) trials on non-artemisininbased drugs were clustered in Africa, whereas trials on artemisinin-based drugs were performed worldwide. The non-artemisinin-based anti-malarials were combined because too few efficacy studies were available to model them independently. Bivariate analyses relating each response variable with all covariates were performed, and the estimated odds ratios (with associated confidence intervals) and Wald's p-values were used to assess the significance of the association. This initial process was performed to remove non-significant variables, as the final variable selection was completed later while fitting the geostatistical model. Pearson's correlation coefficient was used to detect highly correlated covariates. Collinear covariates $(r>0.6)$ were excluded by ranking them based on their goodness-of-fit, assessed through Akaike Information Criteria (AIC), as well as the residual deviance [76], and removing the less predictive covariates of collinear pairs. Further, multicollinearity was checked by assessing the Variance Inflation Factor (VIF) and performing the Farrar-Glauber test [77]. The selected set of covariates was used in the next steps of model development (Additional file 1: Section 3-Table S3.4).

\section{Structure of predictive model}

The analysis utilized a modelling approach that characterized the spatial process and temporal patterns explicitly to derive predictions and estimate uncertainties [4]. The AmE, for location $k$ and year $j, Y_{k j}$, was assumed to follow a binomial distribution denoted $Y_{k j} \sim \operatorname{Binomial}\left(n_{k j}, \theta_{k j}\right)$ where $n_{k j}$ was the sample size (the number of patients involved) and $\theta_{k j}$ was the probability of treatment success. The relation between the estimates of AmE, covariates, and random effects was modelled via the logit link function:

$$
\begin{aligned}
& \operatorname{logit}\left(\theta_{k j}\right)=\ln \left(\frac{\theta_{k j}}{1-\theta_{k j}}\right)=\eta_{k j} \\
& \eta_{k j}=\beta_{0}+\boldsymbol{x}_{k j}^{\top} \boldsymbol{\beta}+\psi_{k}+\xi_{j},
\end{aligned}
$$




$$
\boldsymbol{\beta} \sim N\left(\boldsymbol{\mu}_{\beta}, \boldsymbol{\Sigma}_{\beta}\right)
$$

where $\eta_{k j}$ was the linear predictor, $\beta_{0}$ the intercept, $\boldsymbol{x}_{k j}^{\top}$ was the matrix of covariates and $\boldsymbol{\beta}=\left(\beta_{1}, \beta_{2}, \ldots, \beta_{p}\right)$ was the vector of regression coefficients assumed to follow a zero mean Gaussian distribution, $\boldsymbol{\mu}_{\beta}=0$, and a variance $\boldsymbol{\Sigma}_{\beta} \cdot \psi=\left(\psi_{1}, \ldots \psi_{k}\right)^{T}$ which denotes the spatial random effect which accounted for the spatial dependence. This was assumed to follow a multivariate normal distribution with Matérn covariance function and zero mean (i.e., $\left.\psi \sim M V N\left(0, \sum\right)\right)$. With this specification, the distance at which the spatial correlation is close to 0.1, the spatial range, $r$, and the spatial variance $\sigma^{2}$ were estimated. $\xi_{j} \sim \mathcal{N}\left(\rho \xi_{j-1}, \tau^{2}\right), j>1$ represent the auto-correlated temporal error terms, while $\xi_{1} \sim \mathcal{N}\left(0, \tau^{2}\right) ; \tau$ and $\rho$ are the precision and correlation parameters, respectively. This was a stationary autoregressive process of order one (i.e., AR1), with a Markovian temporal structure, in which the AmE of year $j-1$ influenced the AmE in year $j$. Inclusion of the independent and identically distributed (i.i.d.) site-specific random effects was tested in all model formulations and assessed to see if it improved the fit.

The described model was used to predict AmE, defined as the proportion of individuals with falciparum malaria infection effectively treated with the anti-malarial drug at different places and periods in the context of the actual clinical setting, while accounting for the health system, climate, environment, and socio-demographic factors. Models were fitted in a Bayesian framework using R-INLA [78, 79]. The Bayesian model formulation was completed by assigning a prior distribution to all unknown parameters and hyperparameters. The initial model assumed non-informative priors [78] and was further refined to include weakly informative priors if doing so improved model performance.

\section{Model validation and selection}

A sensitivity analysis was done to examine the choice of priors for the hyperparameters, the likelihood of the outcome and inclusion of space-time interaction. For the temporal component, i.i.d., first-order random walk (RW1), second-order random walk (RW2), and firstorder autoregressive (AR1) models were tested [80]. For the spatial components, range and variance, the effect of a non-informative prior was compared to penalized complexity (PC) priors [81], and a fixed range. Both beta and binomial distributions were considered for the outcome variable. Furthermore, a model with an overall temporal trend common to all spatial units and an overall spatial effect common to all time periods was compared against a model that allowed variation over space and time. Watanabe Akaike information criterion (WAIC), root mean squared errors (RMSE), and the correlation between the observed data and the predicted values, the $\mathrm{R}^{2}$, were used to compare the model fits. Using the variables retained from the first selection process, the spacetime geostatistical model was built sequentially, starting with Model 1 containing no covariates; Model 2 with national level covariates; Model 3 with environmental and climate covariates; and, Model 4 with a combination of environmental, climate and national level covariates. All models included an annual time variable. Models were subjected to validation and calibration procedures using conditional predictive ordinate $(\mathrm{CPO})$ and probability integral transform (PIT) to assess predictive performance [82-87].

Additional out-of-sample prediction was performed using a threefold cross-validation ( $30 / 70$ rule) in which the response data were split randomly into 3 non-overlapping subsets. Two sub-sets were used for model training, while the third sub-set was used for validation. This process was repeated 12 times for 4 different splits of the data. Through this method each observation was used in a test and training set. RMSE and $\mathrm{R}^{2}$, calculated between the observed and predicted data are used to assess model performance. Furthermore, in-sample and out-of-sample estimates of the RMSE were compared to assess overor under-fitting. The statistics for model comparisons, together with results of validation/calibration and predictive ability, were used to select the best model. The models were fitted using 1991-2016 data. To forecast AmE for 2017, 2018 and 2019, the spatial pattern was assumed to be constant after 2016 and thus the pixel-level temporal trends in AmE were interpolated linearly from the time series models. The modelling framework is further explained in Additional file 1: Section 3-Figure S3.1.

\section{Calculation of final AmE estimates and presentation of results}

The estimates obtained from the model were then adjusted for drug quality and patients' adherence. Due to very limited available information on these important factors, uniform values were used for all regions and all time periods. First, based on the review by Ozawa et al., a penalty of $19.1 \%$ was applied to account for the prevalence of sub-standard or falsified antimalarials [88]. Then a $5.0 \%$ penalization was used to account for the imperfect adherence to treatment [66]. To present the spatial distribution, the years between 1991 and 2019 were categorized to 1991-2000, 20012005, 2006-2010, 2011-2015, and 2016-2019. The last period is considered a forecasted period. The year ranges used in this categorization were selected based on temporal clustering of clinical efficacy trials and benchmark years for anti-malarial drug usage, 
including the WHO's recommendation on ACT in 2001, peak of malaria burden in 2005, and high degree of adoption of ACT as first-line treatment in sub-Saharan Africa in 2011. Non-artemisinin-based anti-malarial effectiveness was not considered for the period after 2015 due to few non-artemisinin clinical efficacy trials being conducted after 2010 . The resulting maps present the average AmE levels for the stated periods. Median and interquartile ranges (IQR) are used to report due to the asymmetric distribution of the AmE estimates. To capture uncertainty and spatial heterogeneity in the predicted AmE levels, stochastic realisations from the models' posterior distributions were generated and then summarized to obtain mean and uncertainty estimates for national and sub-national administrative units.

\section{Results}

\section{Study characteristics}

The observed data covered the period 1991-2016. Trials on artemisinin-based anti-malarials (ACT + monotherapy) accounted for $88 \%$ of the studies included in this analysis. Most studies were performed in Africa (69.4\%) and the most commonly tested anti-malarial was artemether-lumefantrine $(\mathrm{n}=169)$ (Table 1$)$. Among countries, Thailand (28 studies, 73 treatments), India (6 studies, 45 treatments), and Uganda (21 studies, 44 treatments) had the most studies on artemisininbased anti-malarials. Uganda (32 treatments) and Madagascar (14 treatments) had the most studies on non-artemisinin-based anti-malarials (Additional file 1: Section 2). Descriptive analysis of all the treatments indicated that efficacy of artemisinin-based anti-malarial drugs remained high in most regions throughout the study period, while that of non-artemisinin-based declined over time. The median levels for artemisininbased drugs obtained from the trials for the periods were 99.3\% (1991-2000), 97.1\% (2001-2005), 98.2\% (2006-2010), and 98.8\% (2011-2016). For the nonartemisinin drugs, these were $83.2 \%$ (1991-2000),
81.5\% (2001-2005), 88.7\% (2006-2010), and 51.6\% (2011-2016).

\section{Model selection}

The final specification of the model, with binomial likelihood and variation over space and time, used noninformative priors for the regression coefficients, PC priors for spatial effects and an AR1 structure for the temporal effects. The in-sample validation and model calibration indicated good performance of the models. Similar findings were obtained for the threefold crossvalidation. The RMSE of the training set did not differ much from that of the validation set indicating that overfitting was not an issue. The $\mathrm{R}^{2}$ between observed and predicted ranged between 0.86 and 0.89 (artemisininbased) and 0.63 and 0.66 (non-artemisinin), depending on the set of covariates used. Model 4 had the best fit, as indicated by the lowest WAIC, and high predictive ability as indicated by high $\mathrm{R}^{2}$ (Table 2). Performance metrics for the final geostatistical models fitted for both drug types are shown in Table 2.

\section{Anti-malarial drug effectiveness}

The AmE of artemisinin-based and non-artemisinin antimalarial drugs was predicted annually for the period 1991-2019 for all malaria-endemic countries then aggregated into multi-year categories as indicated above. High-resolution maps $(5 \times 5 \mathrm{~km})$ of AmE, adjusted for the drug quality and adherence, show the global spatial distribution and country-to-country variations (Figs. 1 and 2). Maps of the corresponding uncertainty measure, the IQR, are shown in Additional file 1: Section 4.

\section{Artemisinin-based anti-malarial drugs}

The global AmE of artemisinin-based drugs was $67.4 \%$ (IQR: 33.3-75.8), 68.8\% (IQR: 39.2-75.8), 70.1\% (IQR: 43.6-76.0), 70.7\% (IQR: 45.5-76.2), and 71.8\% (IQR: 46.9-76.4) for the 1991-2000, 2001-2005, 2006-2010, 2011-2015, and 2016-2019 periods, respectively. Modelled AmE was $29.6 \%$ lower, on average, than the levels obtained from trials, largely due to the adjustments applied for drug quality and patient adherence. The

Table 2 Model assessment and selection in estimation of anti-malarial drug effectiveness

\begin{tabular}{|c|c|c|c|c|c|c|c|c|}
\hline \multirow[t]{2}{*}{ Metric } & \multicolumn{4}{|c|}{ Non- artemisinin anti-malarial drugs } & \multicolumn{4}{|c|}{ Artemisinin-based anti-malarial drugs } \\
\hline & Model 1 & Model 2 & Model 3 & Model 4 & Model 1 & Model 2 & Model 3 & Model 4 \\
\hline WAIC & 3897.03 & 3760.59 & 3757.10 & 3749.13 & 4898.14 & 4855.83 & 4838.53 & 4800.72 \\
\hline RMSE & 0.171 & 0.167 & 0.165 & 0.165 & 0.039 & 0.038 & 0.037 & 0.037 \\
\hline R-squared & 0.635 & 0.654 & 0.664 & 0.665 & 0.86 & 0.87 & 0.88 & 0.89 \\
\hline
\end{tabular}

Model 1-no covariates; Model 2-national level covariates; Model 3-environmental and climate covariates; Model 4-a combination of environmental, climate and national level covariates 


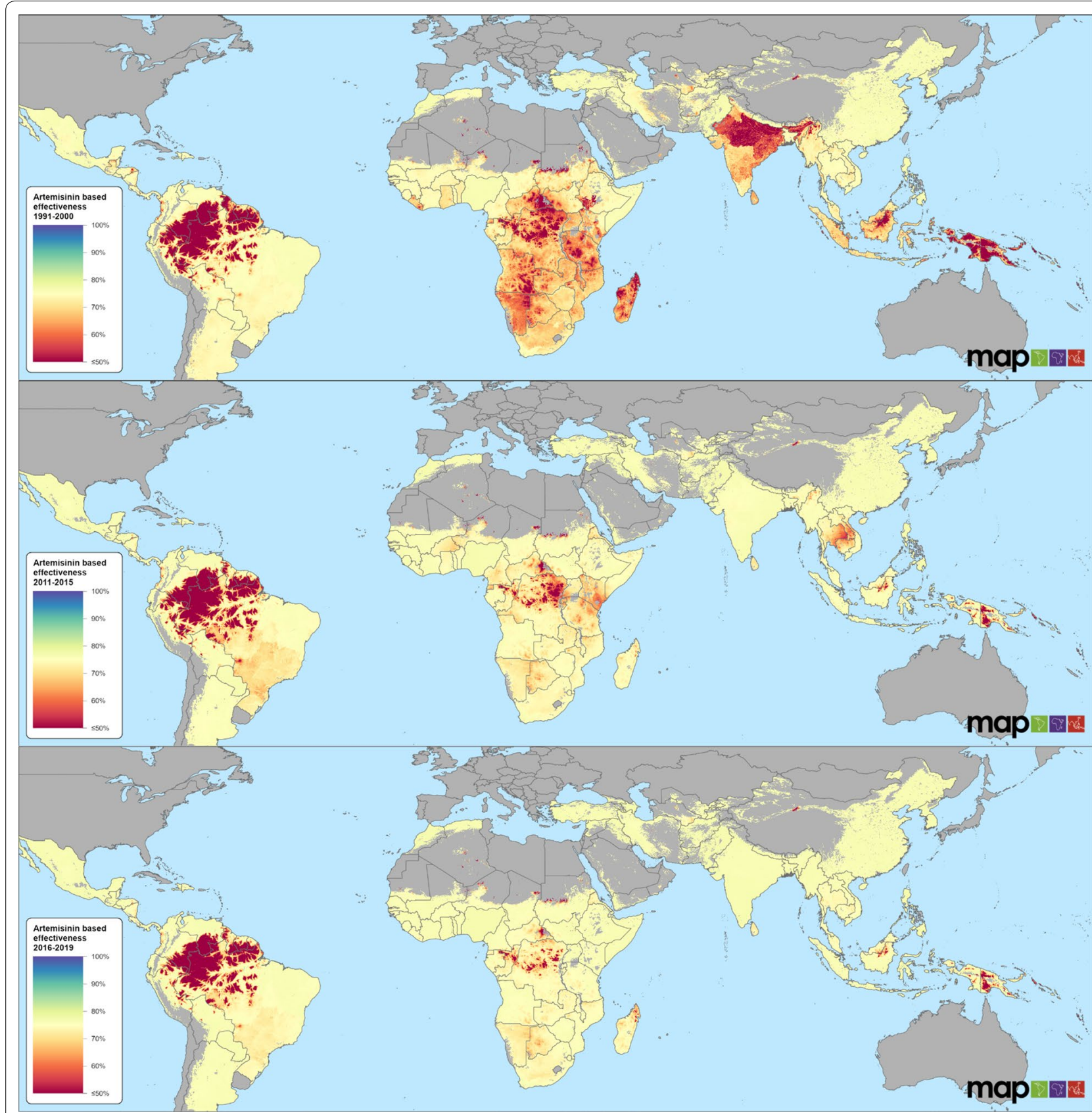

Fig. 1 Spatiotemporal distribution of effectiveness of artemisinin-based anti-malarial drugs for periods 1991-2000, 2011-2015, and 2016-2019. Maps for 2001-2005 and 2006-2010 are presented in Additional file 1: Figure S4.1

relatively stable estimates over time indicated that $\mathrm{AmE}$ of artemisinin-based drugs remained high and increased by $6.5 \%$ from the years before 2000 to those after 2016 (Fig. 1). In the years before 2000, countries in sub-Saharan Africa, South America, India, and on the islands of the Indonesian archipelago were predicted to have lower AmE levels (<60\%) (Fig. 1). The situation improved substantially by the 2011-2015 period, with few remaining pockets of low AmE in sub-Saharan Africa. The levels of AmE dropped in Southeast Asian countries, including Thailand, Myanmar, Cambodia, and Laos. Little improvement in AmE was predicted for P. falciparum-endemic countries in South America including Guyana, Suriname, and parts of Brazil, Venezuela and Colombia. Generally, the model predicts improved AmE for artemisinin-based drugs for the period 2016-2019 (Fig. 1). 


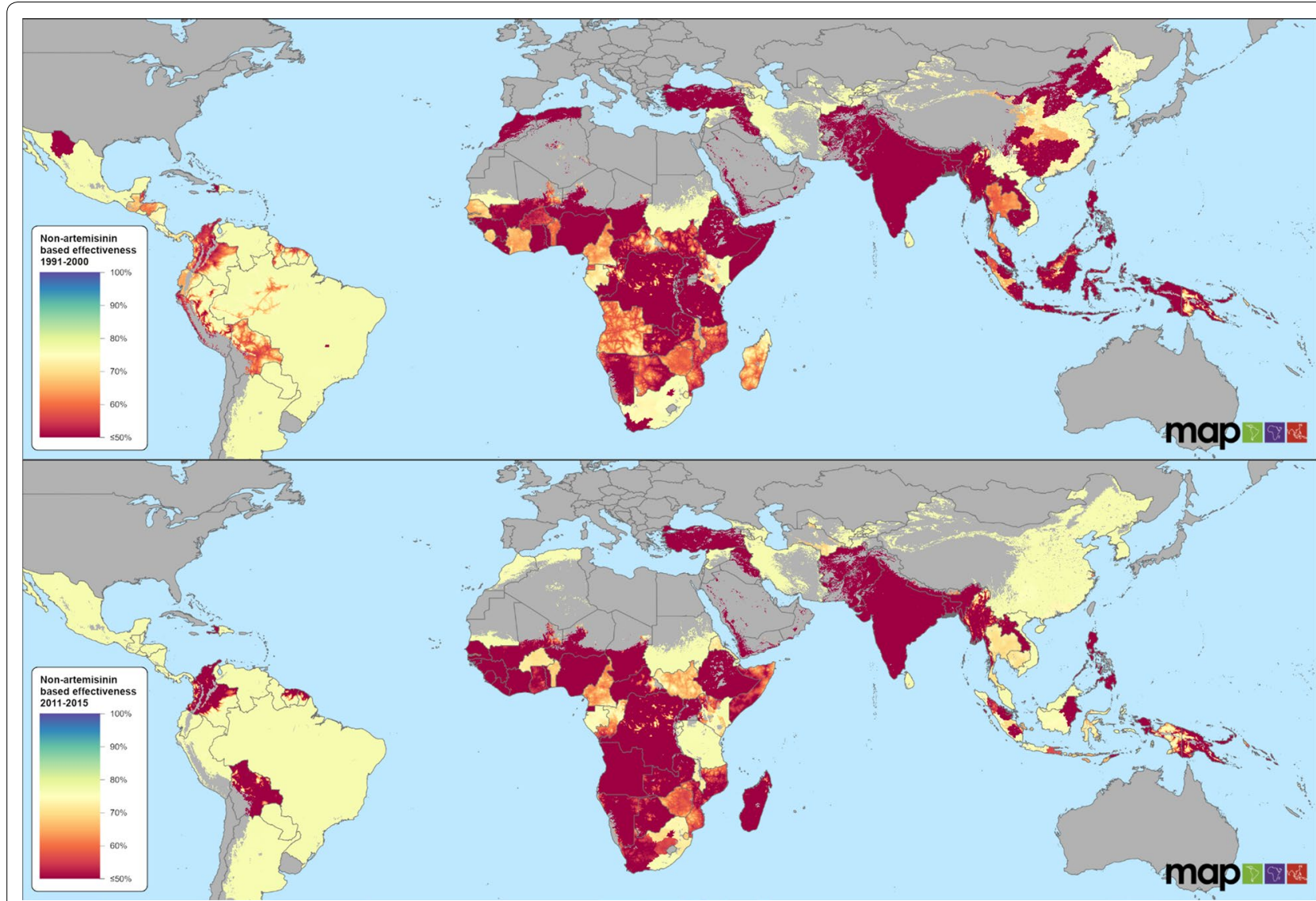

Fig. 2 Spatiotemporal distribution of effectiveness of non-artemisinin anti-malarial drugs for periods 1991-2000 and 2011-2015. Maps for 2001-2005 and 2006-2010 are presented in Additional file 1: Figure S4.2

AmE levels often remained stable in countries where multiple ACT medicine was used as the first-line drug of choice, a practice which became more widespread in the mid-2000s. Noteworthy examples include Ghana, Nigeria, Senegal, Angola, and India. Factors positively related to ACT effectiveness in the analysis include access to health care, human resource capacity (skilled birth attendants as a proxy), education levels, and accessibility to cities. In contrast, AmE dropped in countries including Indonesia, Thailand and Vietnam where Dihydroartemisinin piperaquine (DHAP) was the sole first-line treatment and resistance emerged to either artesunate or the partner drug (Fig. 1).

\section{Non-artemisinin-based anti-malarial drugs}

The global AmE of non-artemisinin-based drugs was much lower than the AmE of artemisinin-based drugs, and these estimates also had higher associated levels of uncertainty. Predicted AmE levels were 52.3\% (IQR: 17.9-74.9), 53.1\% (IQR: 21.6-74.0), 55.9\% (IQR: 22.6-73.5), and 55.5\% (IQR: 27.1-73.4) for the 19912000, 2001-2005, 2006-2010, and 2011-2015 periods, respectively. Predictions for these drugs beyond 2015 were not made due to data limitations. Globally, the AmE levels indicated an increase of $6.1 \%$ from the 1991-2000 period to the 2011-2015 period (Additional file 1: Section 4-Figure S4.2). The predicted AmE levels were approximately $36 \%$ lower than the mean values of measured effectiveness in a controlled trial setting. For all periods, the AmE estimates were lowest in sub-Saharan Africa and in Asia with a few countries observed to regain slight increases in effectiveness after 2011 (Fig. 2). In contrast to patterns elsewhere, the AmE of non-artemisinin-based drugs in Central and South America remained moderate and stable (Fig. 2).

National level covariates strongly affected the predicted effectiveness of non-artemisinin-based antimalarial drugs. Health system and infrastructure factors, including coverage of skilled human resources, universal coverage, health care access, and accessibility to cities, were associated with increasing effectiveness. Factors negatively correlated with effectiveness included high levels of out of pocket expenditure and urbanization. 


\section{Discussion}

This study is the first attempt to produce high-resolution maps of spatiotemporal patterns of AmE, while accounting for the prevalence of sub-standard and falsified antimalarials and patient adherence in all malaria-endemic countries. The resulting temporal patterns highlight changes in AmE from 1991 to 2019 while the spatial patterns illustrate heterogeneity between and within countries. Effectiveness of anti-malarial drugs used for treatment has a direct link to the progress of impact indicators, such as rates of malaria incidence and mortality in the population, which makes knowledge of this metric essential to control programmes. These findings provide additional evidence on practical considerations for implementing malaria treatment policies to ensure adequate anti-malarial effectiveness, including highlighting the roles that drug quality, adherence and health system quality play in AmE. Despite high clinical efficacy levels obtained under controlled conditions, AmE in artemisinin-based drugs dropped by at least a third when applied in the routine care delivery system. The drop was higher for the non-artemisinin-based drugs, likely due to increased level of resistance to those treatments. Overall, the results show that artemisinin-based anti-malarials have higher and more stable AmE compared to non-artemisinin-based drugs. For artemisininbased anti-malarials, areas of lower AmE include the central and eastern part of sub-Saharan Africa, remote areas of South America, and Southeast Asia. The findings from this analysis suggest that non-artemisinin drugs remained effective for uncomplicated falciparum malaria in the South and Central American regions through 2015, but performed poorly elsewhere. However, this finding is associated with high levels of uncertainty due to little clinical efficacy data within the Americas. As such, this result should be viewed cautiously, and careful monitoring of both anti-malarial drug efficacy and health system performance metrics associated with effectiveness should accompany the continued use of non-ACT as front-line treatment in Central American countries. Resistance to non-artemisinin-based drugs has been observed in Africa since the 1980s [89] and inspired treatment policy changes to ACT since 2003 [90]. The WHO critical threshold for clinical efficacy is set at $>90 \%$, but no documented threshold is set for AmE. Further investigation is needed in areas with low predicted effectiveness levels to determine factors driving the gap between the two metrics. Nevertheless, these findings suggest that, despite the availability of efficacious anti-malarials and over $80 \%$ of endemic countries adopting them as first line treatments, policy implementation gaps and challenges remain and impact malaria incidence and mortality. The emergence of resistance of artemisinin derivatives in Southeast Asia and its possible extension in other endemic regions may very negatively impact $\mathrm{AmE}$, repeating patterns observed for non-artemisinin-based drugs in the past.

Time was an important parameter for modelling patterns of artemisinin-based AmE. In the early 2000s ACT was introduced, proved to be highly effective at treating malaria, and thus was adopted by many countries as a first-line drug to treat falciparum malaria. Prior to this period, artemisinin-based monotherapy was widely used, but this approach has since fallen out of favour as it is believed to promote emergence of drug resistance $[14,16]$. In some regions, despite ACT being adopted as first-line treatment policy, their implementation has faced a number of challenges. Demographic and health survey and malaria indicators survey and other literature have shown that health workers still prescribe other anti-malarials for a range of reasons including patient preference, provider perception on specific types of antimalarials, ACT stock-outs, costs including those incurred when accessing care, and higher availability and access to non-recommended anti-malarials. These multifaceted drivers of AmE have been reported across Africa, including in Kenya [91], Cameroon [92], Democratic Republic of Congo (DRC) [93], and Madagascar, and have slowed progress towards increasing AmE and reducing malaria burden [94]. The long term use of single anti-malarial drugs results in high drug-based selective pressure, which has been proven to decrease parasite sensitivity [95], and could explain the decreasing AmE patterns observed in some settings. Some countries introduced multiple ACT as first-line treatment options, which appears to have maintained the high AmE of ACT by reducing drug pressure. These policies also provided treatment choices to patients, which may have increased adherence. Countries adopting this strategy include Angola, Brazil, Burkina Faso, Nigeria, Senegal, Togo, Sierra Leone, China, and Myanmar [12, 96, 97]; countries with low ACT AmE could potentially use this as a mechanism for improving treatment success.

With the exception of Central America, where nonartemisinin-based AmE was higher in 2011-2015 than in 1991-2000, non-artemisinin drugs had reducing effectiveness in most areas and their efficacy changed less over time. This result is supported by the continued use of chloroquine as first-line treatment in several Central American countries [11]. For example, in Belize, Costa Rica, and El Salvador, first-line falciparum malaria treatment consists of chloroquine combined with primaquine (one-day dose) [11]. Using non-artemisinin-based drugs in combination and short-term dosing requirements may have slowed the development of drug resistance, increase patient adherence, efficacy and effectiveness, and led to the patterns observed in the findings (Additional file 1: 
Section 1) [11]. However, these findings are somewhat speculative as very few datasets on performance of anti-malarial drugs were available from Central and South America, resulting in more uncertain estimates. An unexpected finding of this work was a resurgence in non-ACT AmE in places where they have been banned, such as in Malawi [98]. However, as very few clinical trials on non-ACT have been conducted since the widespread adoption of ACT, this finding is driven by model covariates (e.g., improvements in health systems) rather than response data, and should be interpreted cautiously. By aggregating all ACT within a single analysis, direct assessment of known AmE limitations related to the efficacy of the partner drug (e.g., Artesunate-sulfadoxine-pyrimethamine) were not possible. These limitations include administration aspects, such as duration of treatment and the number of tablets in the dose regimens of the partner drugs $[99,100]$. This could be a possible explanation for the lower drug effectiveness observed in Djibouti, Ecuador, India, Pakistan, and Sudan. Some of these countries changed their treatment policies to other ACT, such as Artemether-lumefantrine or DHAP, to increase effectiveness. However, even the most recently developed ACT, DHAP, faces a threat of resistance in some parts of the Greater Mekong Region [101]. Current efforts to sustain effective treatment of falciparum malaria in such areas include introduction of the triple artemisinin-based combination therapy (TACT) [99]. Lack of data on drug resistance prevented this important parameter from being included within the AmE models.

Health system factors are associated with anti-malarial AmE. Access to health care and human resource capacity influence how and which drugs are prescribed and used. With low access to health care, a significant number of cases will not reach the health care system. Such cases will either not receive any treatment or obtain treatment through other sources, the latter of which may result in the use of a non-first-line treatment and failure to record the type, quality and dosage of the drug within official statistics. In areas where drug monitoring is not effective, irrational provision and unregulated use of anti-malarials might be high, including sub-optimal dosage and increased use of falsified, sub-standard, or nonrecommended medicines, all of which may lead to low effectiveness. Health workers' skills and compliance with treatment guidelines, along with drug availability, determine which drugs are provided to patients and whether the treatments are properly managed, both of which are linked to an effective response to drugs [102]. Sociodemographic factors, including education and accessibility to cities, may have effects similar to those of access to health care and the knowledge of both the patient and health care provider. Political and economic upheaval are also likely to impact treatment AmE. For example, since the mid-1990s, the DRC and Central Republic of Africa have experienced high levels of violence, population displacement, and destruction of infrastructure, including health facilities. These factors reduced access to care, increased rates of infection, and led to poorer management [103]. Similarly, outbreaks of Ebola virus and SARSCoV-2 (COVID-19) have shown the ability to devastate or overwhelm health care systems, which may disrupt access to core malaria interventions [41, 104]. The poor state of the health care systems within low-resource settings could explain low estimates of AmE in areas facing political instability despite the adoption of ACT as firstline treatment in these locations. In the Greater Mekong Region, malaria transmission patterns are rapidly evolving and there is vast spatial heterogeneity. International borders where transmission remains high are of particular concern, as these areas have poorer access to health care facilities and malaria surveillance measures [105]. Furthermore, malaria control measures are very hard to establish and implement effectively within highly mobile migrant populations [106-109].

This analysis has several noteworthy limitations that should be considered when evaluating estimates. First, the sparsity of efficacy trial data, particularly outside subSaharan Africa, led to high levels of uncertainty within the AmE result. This outcome stems from the challenges of conducting efficacy trials, which are costly in terms of money, time and effort, and are rarely conducted in regions with no evidence of treatment failure. Results of efficacy trials conducted by countries or the WHO are not systematically published or made available to stakeholders, nor is individual patient data shared which limits standardization of drug efficacy outcomes. Regional circumstances that are known to affect anti-malarial intervention programmes may also prevent clinical trials from being conducted (e.g., chronic warfare has hampered the implementation of malaria control interventions in South Sudan [110]). To mitigate these important data limitations, countries are urged to explore potential mechanisms to utilize routine surveillance systems for continuous assessment of anti-malarial drugs performance. Future analysis utilizing these estimates may benefit from including country-specific primary data related to utilization of health services and anti-malarials. Routine data from countries provides opportunities to refine this estimate iteratively. A second noteworthy limitation is that this study did not utilize patient-level or pharmacokinetic/pharmacodynamic (PK/PD) data. Such data would provide patient and drug information (e.g., parasitaemia levels, status of fever, genetics, drug concentration, and biological processes) that could be used to refine estimates [111]. Third, individuals are included in 
clinical trials only if they meet eligibility criteria, which may result in under-representation of the segments of the population most affected by malaria. This could occur, for example, if clinical trials over-represent adults when children under five years represent the majority of cases. As a result, trial results may not be generalizable to the real-world population. Fourth, due to non-availability of data on adherence and quality of anti-malarials medicine used for treatment, a constant adjustment was applied uniformly over space and time. These metrics are likely to vary, therefore this scaling may over- or underestimate AmE. Fifth, the socio-demographic covariates used in this analysis were modelled using a limited set of predictor variables and are somewhat collinear despite a variable selection process conducted. This could hinder the interpretation of results by producing circularity within downstream assessments of causal relationships between AmE and metrics of national development. While this is worth noting, this is not a critical limitation to the study, but should be considered if these results are used in an analysis with other GBD covariates. Sixth, most of the covariates are modelled products that are associated with uncertainty, which is difficult to fully propagate within modelling frameworks. The noise inherited in the efficacy estimates from the WWARN database is expected to be the largest contributor of uncertainty in the AmE estimates, as suggested by the random effects, and thus uncertainty in the covariates was comparatively minor but could still lead to underestimated uncertainty in the final results. Finally, the uncertainty related to AmE estimates varies across space and time, which is characterized by producing multiple realisations of $\mathrm{AmE}$ for each year. By summarizing these realisations, mean and uncertainty maps are produced and made available for download so that other researchers can propagate these uncertainties through their analyses appropriately.

\section{Conclusions}

This work utilized data from clinical efficacy trials to produce global-scale predictions of anti-malarial drug effectiveness, while also incorporating information on drug quality and patient adherence. Triangulating modelling and policy decision thresholds, these predictions provide new insights and help characterize spatial patterns in effectiveness, which provide evidence-based and geographically explicit guidelines for optimal medication-based malaria control worldwide. Most malariaendemic countries are progressing towards elimination, with anti-malarial drugs playing a key role in driving down malaria burden. Prompt diagnosis and effective treatment of cases remain an important strategy in the management and control of malaria in all endemic countries. As such, understanding the state of anti-malarial drug effectiveness becomes a crucial component. Findings from this study provide evidence that ACT remains highly effective as first-line treatment for uncomplicated falciparum malaria compared to non-artemisinin based anti-malarials. However, the drug efficacy levels reported in clinical settings are unlikely to reflect real-world efficacy. Results obtained from this analysis suggest that strategies used in different countries to sustain the effectiveness of these drugs are working, including the use of multiple options for first-line treatment and combining artemisinin with other anti-malarial drugs that remain effective locally. These findings are relevant for guiding policy decisions on targeted interventions towards malaria and contribute to global malaria burden estimates in the World Malaria Report and the GBD study.

\section{Supplementary information}

Supplementary information accompanies this paper at https://doi. org/10.1186/s12936-020-03446-8.

Additional file 1. Supplementary information on methods, data and findings.

\section{Abbreviations}

ACT: Artemisinin-based combination therapy; AL: Artemether-lumefantrine; AmE: Anti-malarial drug effectiveness; ASAQ: Artesunate-amodiaquine; ASMQ: Artesunate-mefloquine; ASSP: Artesunate-sulfadoxine-pyrimethamine; DHAP: Dihydroartemisinin-piperaquine; GBD: Global burden of disease; IHME: Institute for Health Metrics and Evaluation; IQR: Interquartile range; MAP: Malaria Atlas Project; PC: Penalized complexity; WHO: World Health Organization; WWARN: Worldwide Anti-malarial Resistance Network.

\section{Acknowledgements}

The WorldWide Anti-malarial Resistance Network (WWARN) is thanked for the data provision and technical support to all issues related to the aggregated database.

\section{Authors' contributions}

DJW, PWG and GR designed the original study; GR conducted the literature review; SFR, DJW revised the study design and the analysis plan. KAT, GR, GSH, PJG, HSG ensure the availability of data for analysis; SFR and GR conducted the analysis with technical inputs from DJW, TCDL, AN, AP, MN. SFR and GR drafted the initial version of the manuscript; SHK, JAR and ELC created the visualisations; SFR, GR, PJG, DJW interpreted the findings. All authors read and approved the final manuscript.

\section{Funding}

This work was funded by Bill \& Melinda Gates Foundation Grant No. INV008274/OPP1152978. The funding body had no role in the design of the study and collection, analysis, and interpretation of data and in writing the manuscript.

\section{Availability of data and materials}

The datasets used and/or analysed during the current study are available from the corresponding author on reasonable request.

Ethics approval and consent to participate Not applicable.

Consent for publication Not applicable. 


\section{Competing interests}

The authors declare that they have no competing interests.

\section{Author details}

${ }^{1}$ Malaria Atlas Project, Big Data Institute, Nuffield Department of Medicine, University of Oxford, Oxford, UK. ${ }^{2}$ WorldWide Anti-Malarial Resistance Network (WWARN), Oxford, UK. ${ }^{3}$ Infectious Diseases Data Observatory (IDDO), Oxford, UK. ${ }^{4}$ Centre for Tropical Medicine and Global Health, Nuffield Department of Clinical Medicine, University of Oxford, Oxford, UK. ${ }^{5}$ Institute for Disease Modeling, Bellevue, WA, USA. ${ }^{6}$ Imperial College London, London, UK. ${ }^{7}$ Telethon Kids Institute, Perth, Australia. ${ }^{8}$ Curtin University, Perth, Australia. ${ }^{9}$ Center for Data Science, Zhejiang University, Hangzhou 310058, China.

\section{Received: 7 July 2020 Accepted: 10 October 2020}

Published online: 20 October 2020

\section{References}

1. GBD 2017 Mortality Collaborators. Global, regional, and national agesex-specific mortality for 282 causes of death in 195 countries and territories, 1980-2017: a systematic analysis for the Global Burden of Disease Study 2017. Lancet. 2018;392:1736-88.

2. GBD 2016 Mortality Collaborators. Global, regional, and national age-sex specific mortality for 264 causes of death, 1980-2016: a systematic analysis for the Global Burden of Disease Study 2016. Lancet. 2017;390:1151-210.

3. WHO. World malaria report 2014. Geneva: World Health Organization; 2014.

4. Noor AM, Kinyoki DK, Mundia CW, Kabaria CW, Mutua JW, Alegana VA, et al. The changing risk of Plasmodium falciparum malaria infection in Africa: 2000-10: a spatial and temporal analysis of transmission intensity. Lancet. 2014;383:1739-47.

5. WHO. Key points: World malaria report. Geneva: World Health Organization; 2017.

6. WHO. Chemotherapy of malaria. Report of a WHO scientific group. Geneva: World Health Organization; 1967.

7. WHO. Chemotherapy of malaria. Geneva: Wolrd Health Organization; 1986.

8. WHO. Guidelines for the treatment of malaria. 3rd ed. Geneva: World Health Organization; 2015.

9. CDC. List of Maps. Malaria endemic countries. Atlanta: Centers for Disease Control and Prevention; 2018.

10. WHO. WHO certifies Paraguay malaria-free. Geneva: World Health Organization; 2018.

11. WHO. World malaria report 2019. Geneva: World Health Organization; 2019.

12. WHO. World malaria report 2017. Geneva: World Health Organization; 2017.

13. Samarasekera U. Countries race to contain resistance to key anti-malarial. Lancet. 2009:374:277-80

14. Noedl H, Se Y, Schaecher K, Smith BL, Socheat D, Fukuda MM, et al. Evidence of artemisinin-resistant malaria in western Cambodia. N Engl J Med. 2008;359:2619-20.

15. WHO. Emergence and spread of artemisinin resistance calls for intensified efforts to withdraw oral artemisinin-based monotherapy from the market. Geneva: World Health Organization; 2014.

16. Bloland PB. Drug resistance in malaria. Geneva: World Health Organization; 2001.

17. WHO. Anti-malarial drug efficacy and drug resistance. Geneva: World Health Organization; 2018.

18. White NJ. Preventing anti-malarial drug resistance through combinations. Drug Resist Updat. 1998;1:3-9.

19. Yeung S, Pongtavornpinyo W, Hastings IM, Mills AJ, White NJ. Antimalarial drug resistance, artemisinin-based combination therapy, and the contribution of modeling to elucidating policy choices. Am J Trop Med Hyg. 2004;71(2 Suppl):179-86.

20. Phyo AP, Nkhoma S, Stepniewska K, Ashley EA, Nair S, McGready R, et al. Emergence of artemisinin-resistant malaria on the western border of Thailand: a longitudinal study. Lancet. 2012;379:1960-6.
21. Dondorp AM, Nosten F, Yi P, Das D, Phyo AP, Tarning J, et al. Artemisinin resistance in Plasmodium falciparum malaria. N Engl J Med. 2009:361:455-67.

22. Leang R, Barrette A, Bouth DM, Menard D, Abdur R, Duong S, et al. Efficacy of dihydroartemisinin-piperaquine for treatment of uncomplicated Plasmodium falciparum and Plasmodium vivax in Cambodia, 2008 to 2010. Antimicrob Agents Chemother. 2013;57:818-26.

23. Kyaw MP, Nyunt MH, Chit K, Aye MM, Aye KH, Aye MM, et al. Reduced susceptibility of Plasmodium falciparum to artesunate in southern Myanmar. PLOS ONE. 2013;8:e57689.

24. Ashley EA, Dhorda M, Fairhurst RM, Amaratunga C, Lim P, Suon S, et al. Spread of artemisinin resistance in Plasmodium falciparum malaria. $\mathrm{N}$ Engl J Med. 2014;371:411-23.

25. Tun KM, Imwong M, Lwin KM, Win AA, Hlaing TM, Hlaing T, et al. Spread of artemisinin-resistant Plasmodium falciparum in Myanmar: a cross-sectional survey of the K13 molecular marker. Lancet Infect Dis. 2015;15:415-21.

26. Smith Gueye C, Newby G, Hwang J, Phillips AA, Whittaker M, MacArthur $J R$, et al. The challenge of artemisinin resistance can only be met by eliminating Plasmodium falciparum malaria across the Greater Mekong subregion. Malar J. 2014;13:286.

27. Shah NK, Dhillon GP, Dash AP, Arora U, Meshnick SR, Valecha N. Antimalarial drug resistance of Plasmodium falciparum in India: changes over time and space. Lancet Infect Dis. 2011;11:57-64.

28. Davlantes E, Dimbu PR, Ferreira CM, Florinda Joao M, Pode D, Felix J, et al. Efficacy and safety of artemether-lumefantrine, artesunate-amodiaquine, and dihydroartemisinin-piperaquine for the treatment of uncomplicated Plasmodium falciparum malaria in three provinces in Angola, 2017. Malar J. 2018;17:144.

29. Raman J, Kagoro FM, Mabuza A, Malatje G, Reid A, Frean J, et al. Absence of kelch13 artemisinin resistance markers but strong selection for lumefantrine-tolerance molecular markers following 18 years of artemisinin-based combination therapy use in Mpumalanga Province, South Africa (2001-2018). Malar J. 2019;18:280.

30. Uwimana A, Legrand $\mathrm{E}$, Stokes BH, Ndikumana JM, Warsame M, Umulisa $\mathrm{N}$, et al. Emergence and clonal expansion of in vitro artemisinin-resistant Plasmodium falciparum kelch13 R561H mutant parasites in Rwanda. Nat Med. 2020;3:1-7.

31. Gatton ML, Martin LB, Cheng Q. Evolution of resistance to sulfadoxinepyrimethamine in Plasmodium falciparum. Antimicrob Agents Chemother. 2004:48:2116-23.

32. Holmgren G, Gil JP, Ferreira PM, Veiga MI, Obonyo CO, Bjorkman A Amodiaquine resistant Plasmodium falciparum malaria in vivo is associated with selection of pfcrt 76T and pfmdr $186 \mathrm{Y}$. Infect Genet Evol. 2006;6:309-14

33. WHO. A study on the public health and socioeconomic impact of substandard and falsified medical products. Geneva: World Health Organization; 2017.

34. Risha PG, Shewiyo D, Msami A, Masuki G, Vergote G, Vervaet C, et al. In vitro evaluation of the quality of essential drugs on the Tanzanian market. Trop Med Int Health. 2002;7:701-7.

35. Leslie T, Kaur H, Mohammed N, Kolaczinski K, Ord RL, Rowland M. Epidemic of Plasmodium falciparum malaria involving substandard antimalarial drugs, Pakistan, 2003. Emerg Infect Dis. 2009;15:1753-9.

36. Johnston A, Holt DW. Substandard drugs: a potential crisis for public health. Br J Clin Pharmacol. 2014;78:218-43.

37. Newton PN, Caillet C, Guerin PJ. A link between poor quality antimalarials and malaria drug resistance? Expert Rev Anti Infect Ther 2016:14:531-3.

38. WHO. Methods for surveillance of anti-malarial drug efficacy. Geneva: World Health Organization; 2009.

39. Dahal P, d'Alessandro U, Dorsey G, Guerin PJ, Nsanzabana C, Price RN, et al. Clinical determinants of early parasitological response to ACTs in African patients with uncomplicated falciparum malaria: a literature review and meta-analysis of individual patient data. BMC Med. 2015:13:212.

40. Dondorp AM, Smithuis F, Woodrow C, von Seidlein L. How to contain artemisinin- and multidrug-resistant falciparum malaria. Trends Parasitol. 2017;33:353-63.

41. WHO. Global Technical strategy for malaria 2016-2030. Geneva: World Health Organization; 2014. 
42. Takata J, Sondo P, Humphreys GS, Burrow R, Maguire B, Hossain MS, et al. The WWARN Clinical Trials Publication Library: a live, open-access database of Plasmodium treatment efficacy trials. Am J Trop Med Hyg. 2020;102:359-68.

43. WHO. Global Report on anti-malarial drug efficacy and drug resistance: 2000-2010. Geneva: World Health Organization; 2010.

44. Leang R, Taylor WR, Bouth DM, Song L, Tarning J, Char MC, et al. Evidence of Plasmodium falciparum malaria multidrug resistance to artemisinin and piperaquine in western Cambodia: dihydroartemisininpiperaquine open-label multicenter clinical assessment. Antimicrob Agents Chemother. 2015;59:4719-26.

45. Yeka A, Tibenderana J, Achan J, D'Alessandro U, Talisuna AO. Efficacy of quinine, artemether-lumefantrine and dihydroartemisinin-piperaquine as rescue treatment for uncomplicated malaria in Ugandan children. PLOS ONE. 2013;8:e53772.

46. Mavoko HM, Nabasumba C, da Luz RI, Tinto H, D'Alessandro U, Kambugu A, et al. Efficacy and safety of re-treatment with the same artemisinin-based combination treatment (ACT) compared with an alternative ACT and quinine plus clindamycin after failure of first-line recommended ACT (QUINACT): a bicentre, open-label, phase 3, randomised controlled trial. Lancet Glob Health. 2017;5:e60-8.

47. WHO. Anti-malarial drug combination therapy. Report of a WHO Technical Consultation. Geneva: World Health Organization; 2001.

48. Sibley $\mathrm{CH}$, Ringwald P. A database of anti-malarial drug resistance. Malar J. 2006;5:48

49. WHO. Parasite drug resistance. Geneva: World Health Organization; 2018

50. WHO. Global anti-malarial drug policy database Africa. Geneva: World Health Organization; 2006

51. Khatib RA, Skarbinski J, Njau JD, Goodman CA, Elling BF, Kahigwa E, et al. Routine delivery of artemisinin-based combination treatment at fixed health facilities reduces malaria prevalence in Tanzania: an observational study. Malar J. 2012;11:140.

52. East African Network for Monitoring Anti-malarial Treatment. The efficacy of anti-malarial monotherapies, sulphadoxine-pyrimethamine and amodiaquine in East Africa: implications for sub-regional policy. Trop Med Int Health. 2003;8:860-7.

53. East African Network for Monitoring Anti-malarial Treatment. Monitoring anti-malarial drug resistance within National Malaria Control Programmes: the EANMAT experience. Trop Med Int Health. 2001;6:891-8.

54. PAHO. AMI/RAVREDA. Amazon malaria initiative/Amazon network for surveillance of anti-malarial drug resistance. Ten years of malaria prevention and control in the region. Panama City: Pan American Health Organization; 2011.

55. WHO. Fifth meeting of the Greater Mekong Subregion (GMS) therapeutic efficacy studies (TES) network. Vietnam: World Health Organization; 2017.

56. Nugent $R$, Back E, Beith A. The race against drug resistance. Center for Global Development's Drug Resistance Working Group; 2010.

57. Pisani E, Botchway S. Sharing individual patient and parasite-level data through the WorldWide Anti-malarial Resistance Network platform: a qualitative case study. Wellcome Open Res. 2017;2:63.

58. Price RN, Dorsey G, Ashley EA, Barnes Kl, Baird JK, d'Alessandro U, et al. World Anti-malarial Resistance Network I: clinical efficacy of anti-malarial drugs. Malar J. 2007:6:119.

59. WWARN. Clinical module: data management and statistical analysis plan version 1.2:; 2012

60. Dahal P, Simpson JA, Dorsey G, Guerin PJ, Price RN, Stepniewska K. Statistical methods to derive efficacy estimates of anti-malarials for uncomplicated Plasmodium falciparum malaria: pitfalls and challenges. Malar J. 2017;16:430.

61. WWARN. Clinical Trials Publication Library 2020. https://www.wwarn .org/tools-resources/literature-reviews/wwarn-clinical-trials-publicatio n-library. Accessed 15 Oct 2020.

62. Gupta SK. Intention-to-treat concept: a review. Perspect Clin Res. 2011:2:109-12.

63. McCoy CE. Understanding the intention-to-treat principle in randomized controlled trials. West J Emerg Med. 2017;18:1075-8.

64. Stepniewska K, White NJ. Some considerations in the design and interpretation of anti-malarial drug trials in uncomplicated falciparum malaria. Malar J. 2006:5:127.
65. Singal AG, Higgins PD, Waljee AK. A primer on effectiveness and efficacy trials. Clin Transl Gastroenterol. 2014;5:e45.

66. Challenger JD, Bruxvoort K, Ghani AC, Okell LC. Assessing the impact of imperfect adherence to artemether-lumefantrine on malaria treatment outcomes using within-host modelling. Nat Commun. 2017:8:1373.

67. Gething PW, Casey DC, Weiss DJ, Bisanzio D, Bhatt S, Cameron E, et al. Mapping Plasmodium falciparum mortality in Africa between 1990 and 2015. N Engl J Med. 2016;375:2435-45.

68. MAP. High resolution interactive maps. Oxford: Malaria Atlas Project; 2019. https://malariaatlas.org/explorer/\#/. Accessed 15 Oct 2020.

69. Weiss DJ, Mappin B, Dalrymple U, Bhatt S, Cameron E, Hay SI, et al. Re-examining environmental correlates of Plasmodium falciparum malaria endemicity: a data-intensive variable selection approach. Malar J. 2015;14:68.

70. IHME. Institute of Health Metrics and Evaluation Data 2020. https:// ghdx.healthdata.org/ihme_data. Accessed 15 Oct 2020.

71. MAP. Malaria Covariates Oxford: Malaria Atlas Project; 2020. https:// malariaatlas.org/data-project/covariates/. Accessed 15 Oct 2020.

72. Weiss DJ, Bhatt S, Mappin B, Van Boeckel TP, Smith DL, Hay SI, et al. Air temperature suitability for Plasmodium falciparum malaria transmission in Africa 2000-2012: a high-resolution spatiotemporal prediction. Malar J. 2014;13:171.

73. Hay SI, Guerra CA, Gething PW, Patil AP, Tatem AJ, Noor AM, et al. A world malaria map: Plasmodium falciparum endemicity in 2007. PLoS Med. 2009;6:e1000048.

74. Dalrymple U, Mappin B, Gething PW. Malaria mapping: understanding the global endemicity of falciparum and vivax malaria. BMC Med. 2015;13:140.

75. Weiss DJ, Atkinson PM, Bhatt S, Mappin B, Hay SI, Gething PW. An effective approach for gap-filling continental scale remotely sensed time-series. ISPRS J Photogramm Remote Sens. 2014;98:106-18.

76. Stensgaard AS, Vounatsou P, Onapa AW, Simonsen PE, Pedersen EM, Rahbek $C$, et al. Bayesian geostatistical modelling of malaria and lymphatic filariasis infections in Uganda: predictors of risk and geographical patterns of co-endemicity. Malar J. 2011;10:298.

77. Farrar DE, Glauber RR. Multicollinearity in regression analysis: the problem revisited. Rev Economics Stat. 1967;49:92-107.

78. Rue H, Martino S, Chopin N. Approximate Bayesian inference for latent Gaussian models using integrated nested Laplace approximations. J R Stat Soc B. 2009;71:319-92.

79. Banerjee S, Gelfand AE, Finley AO, Sang H. Gaussian predictive process models for large spatial data sets. J R Stat Soc B. 2008;70:825-48.

80. Blangiardo M, Cameletti M, Baio G, Rue H. Spatial and spatiotemporal models with R-INLA. Spat Spatiotemporal Epidemiol. 2013;7:39-55.

81. Simpson D, Rue H, Riebler A, Martins TG, Sørbye SH. Penalising model component complexity: a principled, practical approach to constructing priors. Stat Sci. 2017;32:1-28.

82. Spiegelhalter DJ, Best NG, Carlin BP, Van Der Linde A. Bayesian measures of model complexity and fit. J R Stat Soc B. 2002;64:583-639.

83. Czado C, Gneiting T, Held L. Predictive model assessment for count data. Biometrics. 2009:65:1254-61.

84. Alegana VA, Atkinson PM, Lourenco C, Ruktanonchai NW, Bosco C, Erbach-Schoenberg EZ, et al. Advances in mapping malaria for elimination: fine resolution modelling of Plasmodium falciparum incidence. Sci Rep. 2016;6:29628.

85. Pettit LI. The conditional predictive ordinate for the normal distribution. J R Statist Soc. 1990;B:175-84.

86. Gelfand AE, Dey DK, Chang H. Model determination using predictive distirbutions with the implementation via sampling-based methods. Stanford: Department of Statistics, Stanford University; 1992.

87. Dawid AP. Statistical theory: the prequential approach (with discussion). J R Statist Soc. 1984;147:278-92.

88. Ozawa S, Evans DR, Bessias S, Haynie DG, Yemeke TT, Laing SK, et al. Prevalence and estimated economic burden of substandard and falsified medicines in low- and middle-income countries: a systematic review and meta-analysis. JAMA Netw Open. 2018;1:e181662.

89. Peters W. Resistance in human malaria IV: 4 -aminoquinolines and multiple resistance. Chemotherapy and drug resistance in malaria. London: London Academic Press; 1987. p. 659-786. 
90. WHO. Position of WHO's Roll Back Malaria Department on malaria treatment policy. Geneva: World Health Organization; 2003.

91. Wasunna B, Zurovac D, Goodman CA, Snow RW. Why don't health workers prescribe ACT? A qualitative study of factors affecting the prescription of artemether-lumefantrine. Malar J. 2008;7:29.

92. Sayang C, Gausseres M, Vernazza-Licht N, Malvy D, Bley D, Millet P. Treatment of malaria from monotherapy to artemisinin-based combination therapy by health professionals in rural health facilities in southern Cameroon. Malar J. 2009:8:174.

93. Ntamabyaliro NY, Burri C, Nzolo DB, Engo AB, Lula YN, Mampunza SM, et al. Drug use in the management of uncomplicated malaria in public health facilities in the Democratic Republic of the Congo. Malar J. 2018:17:189.

94. Rakotonandrasana DH, Tsukahara T, Yamamoto-Mitani N. Anti-malarial drug prescribing by healthcare workers when malaria testing is negative: a qualitative study in Madagascar. Trop Med Health. 2018;46:13.

95. Parker D, Lerdprom R, Srisatjarak W, Yan G, Sattabongkot J, Wood J, et al. Longitudinal in vitro surveillance of Plasmodium falciparum sensitivity to common anti-malarials in Thailand between 1994 and 2010. Malar J. 2012:11:290.

96. WHO. Roll Back Malaria. World malaria report 2005. Geneva: World Health Organization; 2005

97. WHO. World malaria report 2010. Geneva: World Health Organization; 2010.

98. Laufer MK, Thesing PC, Dzinjalamala FK, Nyirenda OM, Masonga $\mathrm{R}$, Laurens $\mathrm{MB}$, et al. A longitudinal trial comparing chloroquine as monotherapy or in combination with artesunate, azithromycin or atovaquone-proguanil to treat malaria. PLoS ONE. 2012;7:e42284.

99. Dini S, Zaloumis S, Cao P, Price RN, Fowkes FJl, van der Pluijm RW, et al. Investigating the efficacy of triple artemisinin-based combination therapies for treating Plasmodium falciparum malaria patients using mathematical modeling. Antimicrob Agents Chemother. 2018:62:e01068-e1118.

100. Nsanzabana C. Resistance to artemisinin combination therapies (ACTs): do not forget the partner drug! Trop Med Infect Dis. 2019;4:26.

101. Amato R, Pearson RD, Almagro-Garcia J, Amaratunga C, Lim P, Suon $S$, et al. Origins of the current outbreak of multidrug-resistant malaria in southeast Asia: a retrospective genetic study. Lancet Infect Dis. 2018;18:337-45.
102. Galactionova K, Tediosi F, de Savigny D, Smith T, Tanner M. Effective coverage and systems effectiveness for malaria case management in sub-Saharan African countries. PLoS ONE. 2015;10:e0127818.

103. UNDP. Human development report 2011. Sustainability and equity: a better future for all. New York: UN Development Programme; 2011.

104. WHO. The potential impact of health service disruptions on the burden of malaria. Geneva: World Health Organization; 2020.

105. Cui L, Cao Y, Kaewkungwal J, Khamsiriwatchara A, Lawpoolsri S, Soe TN, et al. Malaria elimination in the Greater Mekong Subregion: challenges and prospects. Towards malaria elimination-a leap forward. In: Manguin S, Dev V, editors. Towards malaria elimination. InTechOpen Publ; 2018.

106. Cui L, Yan G, Sattabongkot J, Cao Y, Chen B, Chen X, et al. Malaria in the Greater Mekong Subregion: heterogeneity and complexity. Acta Trop. 2012;121:227-39.

107. Hewitt S, Delacollette C, Chavez I. Malaria situation in the Greater Mekong Subregion. Southeast Asian J Trop Med Public Health. 2013;44(Suppl 1):46-72.

108. Parker DM, Carrara VI, Pukrittayakamee S, McGready R, Nosten FH. Malaria ecology along the Thailand-Myanmar border. Malar J. 2015;14:388.

109. Xu J, Liu H. The challenges of malaria elimination in Yunnan Province, People's Republic of China. Southeast Asian J Trop Med Public Health. 2012;43:819-24.

110. Pasquale H, Jarvese M, Julla A, Doggale C, Sebit B, Lual MY, et al. Malaria control in South Sudan, 2006-2013: strategies, progress and challenges, Malar J. 2013;12:374.

111. van den Borne BE, Landewe RB, Goei The HS, Rietveld JH, Zwinderman $\mathrm{AH}$, Bruyn GA, et al. Combination therapy in recent onset rheumatoid arthritis: a randomized double blind trial of the addition of low dose cyclosporine to patients treated with low dose chloroquine. J Rheumatol. 1998;25:1493-8.

\section{Publisher's Note}

Springer Nature remains neutral with regard to jurisdictional claims in published maps and institutional affiliations.
Ready to submit your research? Choose BMC and benefit from:

- fast, convenient online submission

- thorough peer review by experienced researchers in your field

- rapid publication on acceptance

- support for research data, including large and complex data types

- gold Open Access which fosters wider collaboration and increased citations

- maximum visibility for your research: over $100 \mathrm{M}$ website views per year

At BMC, research is always in progress.

Learn more biomedcentral.com/submissions 